Bull. Korean Math. Soc. 45 (2008), No. 4, pp. 729-737

\title{
ON MULTI-JENSEN FUNCTIONS AND JENSEN DIFFERENCE
}

\author{
KRZYSZTOF CiEPliŃSKI
}

ABSTRACT. In this paper we characterize multi-Jensen functions $f: V^{n} \rightarrow$ $W$, where $n$ is a positive integer, $V, W$ are commutative groups and $V$ is uniquely divisible by 2 . Moreover, under the assumption that $f: \mathbb{R} \rightarrow \mathbb{R}$ is Borel measurable, we obtain representation of $f$ (respectively, $f, g, h$ : $\mathbb{R} \rightarrow \mathbb{R}$ ) such that the Jensen difference

$$
2 f\left(\frac{x+y}{2}\right)-f(x)-f(y)
$$

(respectively, the Pexider difference

$$
\left.2 f\left(\frac{x+y}{2}\right)-g(x)-h(y)\right)
$$

takes values in a countable subgroup of $\mathbb{R}$.

\section{Introduction}

In $2005 \mathrm{~W}$. Prager and J. Schwaiger (see [23]) introduced the notion of multi-Jensen functions $f: V^{n} \longrightarrow W(V$ and $W$ being vector spaces over the rationals) with the connection with generalized polynomials. On the other hand, the stability of the Jensen functional equation

$$
2 f\left(\frac{x+y}{2}\right)=f(x)+f(y)
$$

( $f$ satisfying this equation is called a Jensen mapping) was studied by a number of mathematicians (see for instance [20], [10], [17], [16] and [7]).

Speaking of the stability of a functional equation we follow the question of S. Ulam: "when is it true that the solution of an equation differing slightly from a given one, must of necessity be close to the solution of the given equation?" (see [25]). As the words "differing slightly" and "be close" may have various meanings, different kinds of stability can be dealt with (see for instance [15]).

Received February 22, 2008.

2000 Mathematics Subject Classification. Primary 39B72, 39B82, 39A70, 39B22, 39 B52.

Key words and phrases. multi-Jensen function, multi-additive mapping, stability, Jensen difference, Pexider difference. 
In 2006 J.-H. Bae and W.-G. Park (see [1]) gave the general solution the system of equations

$$
\left\{\begin{array}{l}
2 f\left(\frac{x+y}{2}, z\right)=f(x, z)+f(y, z) \\
2 f\left(x, \frac{y+z}{2}\right)=f(x, y)+f(x, z)
\end{array}\right.
$$

where $f: V^{2} \longrightarrow W$ and $V, W$ are vector spaces. Under some additional assumptions they also proved the stability of this system.

Our first goal is to generalize these results. More precisely, generalizing some outcomes from [23] and [1], we obtain the form of multi-Jensen functions in the case when $V, W$ are commutative groups and $V$ is uniquely divisible by 2 . Moreover, we generalize some results from [16] and [1] dealing with the stability in the spirit of P. Gavruta (see [12] and also [21], [22]).

From an example of G. Godini (see [14]) it can be seen that it is not generally true that a function $f: \mathbb{R} \longrightarrow \mathbb{R}$ for which the Cauchy difference

$$
f(x+y)-f(x)-f(y)
$$

belongs to $\mathbb{Z}$ for all $x, y \in \mathbb{R}$ has to be of the form $A+k$, where $A$ is an additive mapping and $k$ takes integer values only. However, such a representation is possible under some regularity condition imposed on $f$. It seems that J. G. van der Corput was the first who gave such a condition (see [9]). Further results and their generalizations (also concerning the Pexider difference

$$
f(x+y)-g(x)-h(y))
$$

were obtained for instance by K. Baron, PL. Kannappan, J. Brzdęk, N. Frantzikinakis, M. Bajger and the author (see [4], [5], [11], [2], and [8]).

Later the Jensen difference

$$
2 f\left(\frac{x+y}{2}\right)-f(x)-f(y)
$$

(see [5], [13], and [19]) as well as the "quadratic" difference

$$
f(x+y)+f(x-y)-2 f(x)-2 f(y)
$$

(see [6] and [18]) were also investigated.

The second aim of this paper is to study the Jensen difference and the Pexider difference

$$
2 f\left(\frac{x+y}{2}\right)-g(x)-h(y)
$$

where $f, g, h: \mathbb{R} \rightarrow \mathbb{R}$. Under the assumption that $f$ is Borel measurable we obtain representation of $f$ (respectively, $f, g$ and $h$ ) with the Jensen (respectively, Pexider) difference taking values in a countable subgroup of $\mathbb{R}$. 


\section{Main results}

\subsection{Multi-Jensen functions}

We follow the notation used in [23].

Denote by $|S|$ the cardinality of the finite set $S$ and put

$$
\mathbf{n}:=\{1, \ldots, n\}, \quad n \in \mathbb{N},
$$

where $\mathbb{N}$ stands for the set of all positive integers.

Let $V, W$ be commutative groups and $V$ be uniquely divisible by 2 . For sets $S, T$ with $S \subseteq T$ and $x=\left(x_{t}\right)_{t \in T} \in V^{T}$, let $x_{S}:=\left.x\right|_{S} \in V^{S}$, i.e., $\left.x\right|_{S}$ is the restriction of the mapping $x: T \longrightarrow V$ to $S$. For $y \in V^{S}$ let $y^{T} \in V^{T}$ be given by

$$
\left(y^{T}\right)_{S}:=y \text { and }\left(y^{T}\right)_{T \backslash S}:=0 .
$$

Let us also recall that a function $f: V^{T} \longrightarrow W$ is called multi-additive or $T$ additive if it is additive in each variable. Similarly, define $f$ to be multi-Jensen or $T$-Jensen if it is a Jensen mapping in each variable.

Since (see Theorem 1.4 in [5]) every Jensen function $f: V \longrightarrow W$ is of the form

$$
f(x)=a(x)+c, \quad x \in V
$$

with, uniquely determined, a $c \in W$ and an additive mapping $a: V \longrightarrow W$, we have the following

Lemma 2.1. Let $V, W$ be commutative groups and $V$ be uniquely divisible by 2. Assume also that $T$ is a set and $f: V^{T} \rightarrow W$. Then $f$ is multi-additive if and only if it is a multi-Jensen mapping such that for any $i \in T, x \in V^{T}$ with $x(i)=0$ we have $f(x)=0$.

Finally, for $T \subseteq S \subseteq \mathbf{n}$ and $y \in V^{S}$ we write $y_{T}^{\mathbf{n}}$ for $\left(y_{T}\right)^{\mathbf{n}}$, i.e., $y_{T}^{\mathbf{n}}=z \in V^{\mathbf{n}}$ with $z_{T}=y_{T}$ and $z_{\mathbf{n} \backslash T}=0$.

Our first theorem characterizes multi-Jensen functions.

Theorem 2.2. Let $V, W$ be commutative groups and $V$ be uniquely divisible by 2. Assume also that $n \in \mathbb{N}$ and $f: V^{\mathbf{n}} \longrightarrow W$. Then $f$ is multi-Jensen if and only if there is a family $\left(M_{S}\right)_{S \subseteq \mathbf{n}}$ of $S$-additive functions such that

$$
f(x)=\sum_{S \subseteq \mathbf{n}} M_{S}\left(x_{S}\right), \quad x \in V^{\mathbf{n}} .
$$

Proof. Assume that $f: V^{\mathbf{n}} \rightarrow W$ is multi-Jensen and put

$$
M_{S}(y)=\sum_{T \subseteq S}(-1)^{|S \backslash T|} f\left(x_{T}^{\mathbf{n}}\right), \quad y \in V^{S}, S \subseteq \mathbf{n},
$$

where $x:=y^{\mathbf{n}}$. We shall show that these mappings are multi-additive.

Fix an $S \subseteq \mathbf{n}$ and note that for every $T \subseteq S$ the mapping $V^{S} \ni y \longmapsto$ $f\left(y_{T}^{\mathbf{n}}\right)=f\left(x_{T}^{\mathbf{n}}\right) \in W$ is multi-Jensen and, in consequence, so is $M_{S}$. Take 
$i \in S, y \in V^{S}$ with $y(i)=0$ and let $x:=y^{\mathbf{n}}$. Clearly, if $T \subset S$ and $i \in S \backslash T$, then $x_{T}^{\mathbf{n}}=x_{T \cup\{i\}}^{\mathbf{n}}$. Therefore,

$$
\begin{aligned}
M_{S}(y) & =\sum_{T \subseteq S, i \in S \backslash T}(-1)^{|S \backslash T|} f\left(x_{T}^{\mathbf{n}}\right)+\sum_{T \subseteq S, i \in S \backslash T}(-1)^{|S \backslash(T \cup\{i\})|} f\left(x_{T \cup\{i\}}^{\mathbf{n}}\right) \\
& =\sum_{T \subseteq S, i \in S \backslash T}(-1)^{|S \backslash T|}\left(f\left(x_{T}^{\mathbf{n}}\right)-f\left(x_{T}^{\mathbf{n}}\right)\right)=0,
\end{aligned}
$$

and the multi-additivity of $M_{S}$ follows from Lemma 2.1 .

Next, note that

$$
\sum_{S \subseteq \mathbf{n}} M_{S}\left(x_{S}\right)=\sum_{S \subseteq \mathbf{n}} \sum_{T \subseteq S}(-1)^{|S \backslash T|} f\left(x_{T}^{\mathbf{n}}\right)=\sum_{T \subseteq \mathbf{n}} \alpha_{T} f\left(x_{T}^{\mathbf{n}}\right),
$$

where

$$
\alpha_{T}:=\sum_{T \subseteq S \subseteq \mathbf{n}}(-1)^{|S \backslash T|}=\sum_{U \subseteq \mathbf{n} \backslash T}(-1)^{|U|} .
$$

But

and therefore

$$
\alpha_{T}= \begin{cases}1, & T=\mathbf{n}, \\
\sum_{l=0}^{|\mathbf{n} \backslash T|}\left(\begin{array}{c}
|\mathbf{n} \backslash T| \\
l
\end{array}\right)(-1)^{l}=0, & T \neq \mathbf{n},\end{cases}
$$

that is (1) holds.

$$
\sum_{S \subseteq \mathbf{n}} M_{S}\left(x_{S}\right)=f\left(x_{\mathbf{n}}^{\mathbf{n}}\right)=f(x), \quad x \in V^{\mathbf{n}},
$$

On the other hand, if the function $f$ is given by (1), where $\left(M_{S}\right)_{S \subseteq \mathbf{n}}$ is a family of $S$-additive mappings, then the fact that it is multi-Jensen follows from obvious fact that for every $S \subseteq \mathbf{n}$ so is the function $V^{\mathbf{n}} \ni x \longmapsto M_{S}\left(x_{S}\right) \in$ $W$.

\subsection{Stability}

Our next theorem generalizes Theorem 6 from [1] (see also Theorems 1 and 2 and Corollary 3 in [16]).

Theorem 2.3. Let $V$ be a commutative group uniquely divisible by 2 and $W$ be a Banach space. Assume also that $n \in \mathbb{N}$ and for every $i \in \mathbf{n}, \varphi_{i}: V^{n+1} \rightarrow$ $[0, \infty)$ is a mapping such that

$$
\begin{aligned}
& \widetilde{\varphi}_{i}\left(x_{1}, \ldots, x_{n+1}\right) \\
:= & \sum_{j=0}^{\infty} \frac{1}{3^{j+1}}\left[\varphi_{i}\left(3^{j} x_{1}, x_{2}, \ldots, x_{n+1}\right)+\cdots\right. \\
& +\varphi_{i}\left(x_{1}, \ldots, x_{i-2}, 3^{j} x_{i-1}, x_{i}, \ldots, x_{n+1}\right) \\
& +\varphi_{i}\left(x_{1}, \ldots, x_{i-1}, 3^{j} x_{i}, 3^{j} x_{i+1}, x_{i+2}, \ldots, x_{n+1}\right) \\
& +\varphi_{i}\left(x_{1}, \ldots, x_{i+1}, 3^{j} x_{i+2}, x_{i+3}, \ldots, x_{n+1}\right)+\cdots \\
& \left.+\varphi_{i}\left(x_{1}, \ldots, x_{n}, 3^{j} x_{n+1}\right)\right]<\infty, \quad\left(x_{1}, \ldots, x_{n+1}\right) \in V^{n+1} .
\end{aligned}
$$


If $f: V^{n} \longrightarrow W$ is a function satisfying

$$
\begin{aligned}
& \| 2 f\left(x_{1}, \ldots, x_{i-1}, \frac{x_{i}+x_{i}^{\prime}}{2}, x_{i+1}, \ldots, x_{n}\right)-f\left(x_{1}, \ldots, x_{n}\right) \\
& -f\left(x_{1}, \ldots, x_{i-1}, x_{i}^{\prime}, x_{i+1}, \ldots, x_{n}\right) \| \\
\leq & \varphi_{i}\left(x_{1}, \ldots, x_{i}, x_{i}^{\prime}, x_{i+1}, \ldots, x_{n}\right), \\
& \left(x_{1}, \ldots, x_{i}, x_{i}^{\prime}, x_{i+1}, \ldots, x_{n}\right) \in V^{n+1}, i \in \mathbf{n},
\end{aligned}
$$

then for every $i \in \mathbf{n}$ there exists a multi-Jensen mapping $F_{i}: V^{n} \rightarrow W$ for which

$$
\begin{aligned}
& \left\|f\left(x_{1}, \ldots, x_{n}\right)-f\left(x_{1}, \ldots, x_{i-1}, 0, x_{i+1}, \ldots, x_{n}\right)-F_{i}\left(x_{1}, \ldots, x_{n}\right)\right\| \\
& (5) \leq \widetilde{\varphi}_{i}\left(x_{1}, \ldots, x_{i},-x_{i}, x_{i+1}, \ldots, x_{n}\right) \\
& \quad+\widetilde{\varphi}_{i}\left(x_{1}, \ldots, x_{i-1},-x_{i}, 3 x_{i}, x_{i+1}, \ldots, x_{n}\right), \quad\left(x_{1}, \ldots, x_{n}\right) \in V^{n} .
\end{aligned}
$$

For every $i \in \mathbf{n}$ the function $F_{i}$ is given by

$$
\begin{aligned}
& F_{i}\left(x_{1}, \ldots, x_{n}\right) \\
:= & \lim _{j \rightarrow \infty} \frac{1}{3^{j}} f\left(x_{1}, \ldots, x_{i-1}, 3^{j} x_{i}, x_{i+1}, \ldots, x_{n}\right),\left(x_{1}, \ldots, x_{n}\right) \in V^{n} .
\end{aligned}
$$

Proof. Fix $x_{1}, \ldots, x_{n} \in V$ and $i \in \mathbf{n}$. By (4) we get

$$
\begin{aligned}
& \| 2 f\left(x_{1}, \ldots, x_{i-1}, 0, x_{i+1}, \ldots, x_{n}\right)-f\left(x_{1}, \ldots, x_{n}\right) \\
& \quad-f\left(x_{1}, \ldots, x_{i-1},-x_{i}, x_{i+1}, \ldots, x_{n}\right) \| \\
\leq & \varphi_{i}\left(x_{1}, \ldots, x_{i},-x_{i}, x_{i+1}, \ldots, x_{n}\right)
\end{aligned}
$$

and

$$
\begin{aligned}
& \| 2 f\left(x_{1}, \ldots, x_{n}\right)-f\left(x_{1}, \ldots, x_{i-1},-x_{i}, x_{i+1}, \ldots, x_{n}\right) \\
& \quad-f\left(x_{1}, \ldots, x_{i-1}, 3 x_{i}, x_{i+1}, \ldots, x_{n}\right) \| \\
\leq & \varphi_{i}\left(x_{1}, \ldots, x_{i-1},-x_{i}, 3 x_{i}, x_{i+1}, \ldots, x_{n}\right) .
\end{aligned}
$$

Hence

$$
\begin{aligned}
& \quad \| 3 f\left(x_{1}, \ldots, x_{n}\right)-f\left(x_{1}, \ldots, x_{i-1}, 3 x_{i}, x_{i+1}, \ldots, x_{n}\right) \\
& \quad-2 f\left(x_{1}, \ldots, x_{i-1}, 0, x_{i+1}, \ldots, x_{n}\right) \| \\
& \leq \varphi_{i}\left(x_{1}, \ldots, x_{i},-x_{i}, x_{i+1}, \ldots, x_{n}\right) \\
& \quad+\varphi_{i}\left(x_{1}, \ldots, x_{i-1},-x_{i}, 3 x_{i}, x_{i+1}, \ldots, x_{n}\right)
\end{aligned}
$$


and consequently for any non-negative integers $l$ and $m$ such that $l<m$ we obtain

$$
\begin{aligned}
& \| \frac{1}{3^{l}} f\left(x_{1}, \ldots, x_{i-1}, 3^{l} x_{i}, x_{i+1}, \ldots, x_{n}\right) \\
& \quad-\frac{1}{3^{m}} f\left(x_{1}, \ldots, x_{i-1}, 3^{m} x_{i}, x_{i+1}, \ldots, x_{n}\right) \\
& \quad-\sum_{j=l}^{m-1} \frac{2}{3^{j+1}} f\left(x_{1}, \ldots, x_{i-1}, 0, x_{i+1}, \ldots, x_{n}\right) \| \\
& \leq \sum_{j=l}^{m-1} \frac{1}{3^{j+1}}\left[\varphi_{i}\left(x_{1}, \ldots, x_{i-1}, 3^{j} x_{i},-3^{j} x_{i}, x_{i+1}, \ldots, x_{n}\right)\right. \\
& \left.\quad+\varphi_{i}\left(x_{1}, \ldots, x_{i-1},-3^{j} x_{i}, 3^{j+1} x_{i}, x_{i+1}, \ldots, x_{n}\right)\right] .
\end{aligned}
$$

Therefore from (3) it follows that $\left(\frac{1}{3^{j}} f\left(x_{1}, \ldots, x_{i-1}, 3^{j} x_{i}, x_{i+1}, \ldots, x_{n}\right)\right)_{j \in \mathbb{N}}$ is a Cauchy sequence. Since the space $W$ is complete, this sequence is convergent and we define $F_{i}: V^{n} \longrightarrow W$ by (6). Putting $l=0$, letting $m \longrightarrow \infty$ in (7) and using (3) we see that (5) holds.

Finally, fix $x_{i}^{\prime} \in V, j \in \mathbb{N}$ and note that according to (4) we have

$$
\begin{gathered}
\| \frac{2}{3^{j}} f\left(x_{1}, \ldots, x_{i-1}, 3^{j} \frac{x_{i}+x_{i}^{\prime}}{2}, x_{i+1}, \ldots, x_{n}\right) \\
\quad-\frac{1}{3^{j}} f\left(x_{1}, \ldots, x_{i-1}, 3^{j} x_{i}, x_{i+1}, \ldots, x_{n}\right) \\
\quad-\frac{1}{3^{j}} f\left(x_{1}, \ldots, x_{i-1}, 3^{j} x_{i}^{\prime}, x_{i+1}, \ldots, x_{n}\right) \| \\
\leq \frac{1}{3^{j}} \varphi_{i}\left(x_{1}, \ldots, x_{i-1}, 3^{j} x_{i}, 3^{j} x_{i}^{\prime}, x_{i+1}, \ldots, x_{n}\right) .
\end{gathered}
$$

Next, fix $k \in \mathbf{n} \backslash\{i\}, x_{k}^{\prime} \in V$ and assume that $k<i$ (the same arguments apply to the case where $k>i$ ). From (4) it follows that

$$
\begin{aligned}
& \| \frac{2}{3^{j}} f\left(x_{1}, \ldots, x_{k-1}, \frac{x_{k}+x_{k}^{\prime}}{2}, x_{k+1}, \ldots, x_{i-1}, 3^{j} x_{i}, x_{i+1}, \ldots, x_{n}\right) \\
& \quad-\frac{1}{3^{j}} f\left(x_{1}, \ldots, x_{i-1}, 3^{j} x_{i}, x_{i+1}, \ldots, x_{n}\right) \\
& \quad-\frac{1}{3^{j}} f\left(x_{1}, \ldots, x_{k-1}, x_{k}^{\prime}, x_{k+1}, \ldots, x_{i-1}, 3^{j} x_{i}, x_{i+1}, \ldots, x_{n}\right) \| \\
& \leq \frac{1}{3^{j}} \varphi_{k}\left(x_{1}, \ldots, x_{k}, x_{k}^{\prime}, x_{k+1}, \ldots, x_{i-1}, 3^{j} x_{i}, x_{i+1}, \ldots, x_{n}\right) .
\end{aligned}
$$

Letting $j \rightarrow \infty$ in the above inequalities and using (3) we see that the mapping $F_{i}$ is multi-Jensen. 


\subsection{Jensen and Pexider differences}

The results of this section correspond to some outcomes from [13], [19] and [5] (see also Theorem in [11] and Theorem 2 in [8]). The first one deals with the Jensen difference.

Theorem 2.4. Let $E$ be a countable subgroup of $\mathbb{R}$. If $f: \mathbb{R} \longrightarrow \mathbb{R}$ satisfies

$$
2 f\left(\frac{x+y}{2}\right)-f(x)-f(y) \in E, \quad x, y \in \mathbb{R}
$$

and $f$ is Borel measurable, then there exists a $c \in \mathbb{R}$ such that

$$
f(x)-f(0)-c x \in E, \quad x \in \mathbb{R} .
$$

Proof. Put

$$
\widehat{f}(x):=f(x)-f(0), \quad x \in \mathbb{R} .
$$

Then from (8) it follows that

$$
2 \widehat{f}\left(\frac{x+y}{2}\right)-\widehat{f}(x)-\widehat{f}(y) \in E, \quad x, y \in \mathbb{R},
$$

whence, setting $y:=0$, we get

$$
2 \widehat{f}\left(\frac{x}{2}\right)-\widehat{f}(x) \in E, \quad x \in \mathbb{R} .
$$

These two relations give

$$
\begin{aligned}
& \widehat{f}(x+y)-\widehat{f}(x)-\widehat{f}(y) \\
= & 2 \widehat{f}\left(\frac{x+y}{2}\right)-\widehat{f}(x)-\widehat{f}(y)+\widehat{f}(x+y)-2 \widehat{f}\left(\frac{x+y}{2}\right) \in E, \quad x, y \in \mathbb{R} .
\end{aligned}
$$

Since the mapping $\widehat{f}$ is Borel measurable, our assertion follows from Theorem in [11].

We finish with a theorem concerning the Pexider difference.

Theorem 2.5. Let $E$ be a countable subgroup of $\mathbb{R}$. If $f, g, h: \mathbb{R} \rightarrow \mathbb{R}$ satisfy

$$
2 f\left(\frac{x+y}{2}\right)-g(x)-h(y) \in E, \quad x, y \in \mathbb{R}
$$

and $f$ is Borel measurable, then there exists a $c \in \mathbb{R}$ such that

$$
\left\{\begin{array}{l}
2 f(x)-2 f(0)-2 c x \in E, \\
g(x)-g(0)-c x \in E, \quad x \in \mathbb{R} . \\
h(x)-h(0)-c x \in E,
\end{array}\right.
$$


Proof. Put

$$
\widehat{f}(x):=f(x)-f(0), \quad \widehat{g}(x):=g(x)-g(0), \quad \widehat{h}(x):=h(x)-h(0), \quad x \in \mathbb{R} .
$$

Then from (9) it follows that

$$
2 \widehat{f}\left(\frac{x+y}{2}\right)-\widehat{g}(x)-\widehat{h}(y) \in E, \quad x, y \in \mathbb{R} .
$$

Set

$$
\widetilde{f}(x):=2 \widehat{f}\left(\frac{x}{2}\right), \quad x \in \mathbb{R}
$$

and note that in view of (11) we have

$$
\widetilde{f}(x+y)-\widehat{g}(x)-\widehat{h}(y) \in E, \quad x, y \in \mathbb{R} .
$$

Putting $y:=0$ and $x:=0$ in (12) separately we see that

$$
\begin{cases}\widetilde{f}(x)-\widehat{g}(x) \in E, & x \in \mathbb{R}, \\ \widetilde{f}(y)-\widehat{h}(y) \in E, & y \in \mathbb{R} .\end{cases}
$$

(12) and (13) give

$$
\begin{aligned}
& \widetilde{f}(x+y)-\widetilde{f}(x)-\widetilde{f}(y) \\
= & \widetilde{f}(x+y)-\widehat{g}(x)-\widehat{h}(y)+\widehat{g}(x)-\widetilde{f}(x)+\widehat{h}(y)-\widetilde{f}(y) \in E, \quad x, y \in \mathbb{R} .
\end{aligned}
$$

Since the mapping $\tilde{f}$ is Borel measurable, from Theorem in [11] it follows that there is a $c \in \mathbb{R}$ such that $\tilde{f}(x)-c x \in E$ for $x \in \mathbb{R}$. This together with the definition of $\widetilde{f}$ and (13) finishes the proof.

Note added in proof. Recently Hyers-Ulam stability of the multi-Jensen equation in the case when $V, W$ are vector spaces over the rationals was investigated by W. Prager and J. Schwaiger (see [24]).

\section{References}

[1] J.-H. Bae and W.-G. Park, On the solution of a bi-Jensen functional equation and its stability, Bull. Korean Math. Soc. 43 (2006), no. 3, 499-507.

[2] M. Bajger, On the composite Pexider equation modulo a subgroup, Publ. Math. Debrecen 64 (2004), no. 1-2, 39-61.

[3] K. Baron, Orthogonality and additivity modulo a discrete subgroup, Aequationes Math. 70 (2005), no. 1-2, 189-190.

[4] K. Baron and Pl. Kannappan, On the Pexider difference, Fund. Math. 134 (1990), no. 3, 247-254.

[5] J. Brzdęk, The Cauchy and Jensen diferences on semigroups, Publ. Math. Debrecen 48 (1996), no. 1-2, 117-136.

[6] _ On orthogonally exponential functionals, Pacific J. Math. 181 (1997), no. 2, $247-267$.

[7] L. Cadariu and V. Radu, Fixed points and the stability of Jensen's functional equation, JIPAM. J. Inequal. Pure Appl. Math. 4 (2003), no. 1, Article 4.

[8] K. Ciepliński, On a generalized Pexider equation and the Pexider difference, Iteration theory (ECIT '06), 27-36, Grazer Math. Ber., 351, Karl-Franzens-Univ. Graz, Graz, 2007. 
[9] J. G. van der Corput, Goniometrische functies gekarakteriseerd door een functionaal betrekking, Euclides 17 (1940), 55-75.

[10] G. L. Forti, Hyers-Ulam stability of functional equations in several variables, Aequationes Math. 50 (1995), no. 1-2, 143-190.

[11] N. Frantzikinakis, Additive functions modulo a countable subgroup of $\mathbb{R}$, Colloq. Math. 95 (2003), no. 1, 117-122.

[12] P. Gavruta, A generalization of the Hyers-Ulam-Rassias stability of approximately additive mappings, J. Math. Anal. Appl. 184 (1994), no. 3, 431-436.

[13] P. Gavruta, S.-M. Jung, and K.-S. Lee, Remarks on the Pexider equations modulo a subgroup, Far East J. Math. Sci. (FJMS) 19 (2005), no. 2, 215-222.

[14] G. Godini, Set-valued Cauchy functional equation, Rev. Roumaine Math. Pures Appl. 20 (1975), no. 10, 1113-1121.

[15] D. H. Hyers, G. Isac, and Th. M. Rassias, Stability of functional equations in several variables, Progress in Nonlinear Differential Equations and their Applications, 34. Birkhäser Boston, Inc., Boston, MA, 1998.

[16] K.-W. Jun and Y.-H. Lee, A generalization of the Hyers-Ulam-Rassias stability of Jensen's equation, J. Math. Anal. Appl. 238 (1999), no. 1, 305-315.

[17] S.-M. Jung, Hyers-Ulam-Rassias stability of Jensen's equation and its application, Proc. Amer. Math. Soc. 126 (1998), no. 11, 3137-3143.

[18] _ On the quadratic functional equation modulo a subgroup, Indian J. Pure Appl. Math. 36 (2005), no. 8, 441-450.

[19] S.-M. Jung and K.-S. Lee, On the Jensen functional equation modulo a subgroup, J. Appl. Algebra Discrete Struct. 5 (2007), no. 1, 21-32.

[20] Z. Kominek, On a local stability of the Jensen functional equation, Demonstratio Math. 22 (1989), no. 2, 499-507.

[21] A. Najati, Hyers-Ulam-Rassias stability of a cubic functional equation, Bull. Korean Math. Soc. 44 (2007), no. 4, 825-840.

[22] C. Park and Th. M. Rassias, d-isometric linear mappings in linear d-normed Banach modules, J. Korean Math. Soc. 45 (2008), no. 1, 249-271.

[23] W. Prager and J. Schwaiger, Multi-affine and multi-Jensen functions and their connection with generalized polynomials, Aequationes Math. 69 (2005), no. 1-2, 41-57.

[24] _ Stability of the multi-Jensen equation, Bull. Korean Math. Soc. 45 (2008), no. $1,133-142$

[25] S. M. Ulam, Problems in Modern Mathematics, Science Editions John Wiley \& Sons, Inc., New York, 1964.

INSTITUTE OF MATHEMATICS

Pedagogical University

PODCHORA̧ŻYYCH 2

30-084 Kraków, Poland

E-mail address: kc@ap.krakow.pl 